\title{
Myeloid-related protein-14 deficiency promotes inflammation in staphylococcal pneumonia
}

\author{
Ahmed Achouiti, ${ }^{1,2}$, Thomas Vogl ${ }^{3}$, Anne J. Van der Meer ${ }^{1,2}$, Ingrid Stroo ${ }^{1,2}$, \\ Sandrine Florquin ${ }^{4}$, Onno J. de Boer ${ }^{4}$, Johannes Roth ${ }^{3}$, Sacha Zeerleder ${ }^{5,6}$, \\ Cornelis van 't Veer ${ }^{1,2}$, Alex F. de Vos ${ }^{1,2}$ and Tom van der Poll ${ }^{1,2,7}$
}

Affiliations: ${ }^{1}$ Center for Experimental and Molecular Medicine, Academic Medical Center, University of Amsterdam, Amsterdam, The Netherlands. ${ }^{2}$ Center for Infection and Immunity Amsterdam, Academic Medical Center, University of Amsterdam, Amsterdam, The Netherlands. ${ }^{3}$ Institute of Immunology, University of Munster, Munster, Germany. ${ }^{4}$ Dept of Pathology, Academic Medical Center, University of Amsterdam, Amsterdam, The Netherlands. ${ }^{5}$ Dept of Hematology, Academic Medical Center, University of Amsterdam, Amsterdam, The Netherlands. ${ }^{6}$ Dept of Immunopathology, Sanquin Research, Amsterdam, The Netherlands. ${ }^{7}$ Division of Infectious Diseases, Academic Medical Center, University of Amsterdam. Amsterdam, The Netherlands.

Correspondence: Ahmed Achouiti, Academic Medical Center, Center for Experimental and Molecular Medicine, Meibergdreef 9, Room G2-130, 1105 AZ Amsterdam, The Netherlands. E-mail: a.achouitidamc.nl

ABSTRACT Staphylococcus aureus has evolved as an important cause of pneumonia in both hospital and community settings. Staphylococcal lung infection can lead to overwhelming pulmonary inflammation. During infection, neutrophils release complexes of myeloid-related protein (MRP)8 and MRP14 (MRP8/ 14). MRP8/14 has been shown to exert pro-inflammatory and chemotactic activity, and to assist in the killing of $S$. aureus. In the current study we sought to determine the role of MRP8/14 in the host response during $S$. aureus pneumonia.

Pneumonia was induced in wildtype and MRP14-deficient mice (mice unable to form MRP8/14) by intranasal inoculation of $1 \times 10^{7} \mathrm{CFU}$ of $S$. aureus USA300. Mice were sacrificed at $6,24,48$ or $72 \mathrm{~h}$ after infection for analyses.

S. aureus pneumonia was associated with a strong rise in MRP8/14 in bronchoalveolar lavage fluid and lung tissue. Surprisingly, MRP14 deficiency had a limited effect on bacterial clearance and was associated with increased cytokine levels in bronchoalveolar lavage fluid and aggravated lung histopathology. MRP14 deficiency in addition was associated with a diminished transmigration of neutrophils into bronchoalveolar lavage fluid at late time-points after infection together with reduced release of nucleosomes.

MRP8/14 serves in an unexpected protective role for the lung in staphylococcal pneumonia.

@ERSpublications

MRP8/14 unexpectedly protects against excessive pulmonary inflammation in murine staphylococcal pneumonia http://ow.ly/IwYt6

Received: Oct 052014 | Accepted after revision: Jan 04 2015 | First published online: March 182015

Support statement: This work is supported by a grant from the Landsteiner Foundation for Blood transfusion Research (project LSBR 0706).

Conflict of interest: None declared.

Copyright OERS 2015 


\section{Introduction}

In the past few decades, methicillin-resistant Staphylococcus aureus (MRSA) has evolved as the most important cause of nosocomial infections, including hospital-acquired and ventilator-associated pneumonia [1, 2]. In recent years, virulent strains of community-associated MRSA have also emerged, causing infections in individuals without recognised risk factors [1,3]. While these strains are mainly related to skin and soft tissue infections, they may cause more serious clinical presentations, such as fulminant necrotising pneumonia [3-5]. Indeed, infection of the lower respiratory tract by MRSA has an acute onset and can lead to overwhelming pulmonary inflammation contributing to organ damage and high fatality rates [4-7]. Progressive antibiotic resistance could make staphylococcal pneumonia an even larger health threat in the future [1], urging the need to gain more insight into host defence mechanisms that could influence the outcome of this disease.

Acute $S$. aureus infection is associated with a variety of pro-inflammatory mechanisms to massively recruit neutrophils into the lung parenchyma $[8,9]$. In addition to phagocytosis of invaded microorganisms [10], these cells are stimulated to release constitutively available endogenous molecules (also known as alarmins) that are recognised by pattern recognition receptors and are able to perpetuate inflammatory responses [11]. Among these alarmins are complexes of myeloid-related protein (MRP)8 and MRP14 (MRP8/14 known as calprotectin) [12]. Extracellular MRP8/14 induces a variety of innate immune responses including the enhancement of cytokine release via Toll-like receptor (TLR) 4 in response to lipopolysaccharide [13] and leukocyte recruitment $[14,15]$.

Next to its pro-inflammatory effects, MRP8/14 reduces microbial growth in infectious environments by virtue of its metal chelating properties [16]. In recent studies it was shown that MRP8/14 inhibited staphylococcal growth and virulence through the binding of zinc and manganese [16-18]. In accordance, mice deficient for MRP8/14 showed an impaired defence after intravenous S. aureus injection [17, 18]. The antimicrobial properties of MRP8/14 may be facilitated by neutrophil extracellular traps (NETs) [19]. We recently demonstrated that blocking MRP8/14 in NETs, reduced NET-mediated growth inhibition of staphylococci in vitro [20].

In the current study we investigated the role of MRP8/14 in inflammation and bacterial clearance in experimentally induced staphylococcal pneumonia. To this end, we used wildtype and MRP14-deficient $\left(m r p 14^{-1-}\right)$ mice that were infected with a strain of community associated MRSA (USA300) via the airways. The $m r p 14^{-/-}$mice lack MRP8 at a protein level, despite normal MRP8 mRNA levels and are, therefore, deficient for MRP8/14 [13]. We hypothesised that $m r p 14^{-/}$mice would demonstrate an impaired clearance of staphylococci from the lungs but potentially with attenuated associated inflammation. Surprisingly, in the study reported here, $m r p 14^{-/-}$mice displayed only minimally enhanced bacterial loads in the lungs compared to wildtype mice, with enhanced pulmonary cytokine levels and aggravated lung histopathology.

\section{Methods}

Mice

C57Bl/6 wildtype mice were purchased from Charles River Laboratories Inc. (Maastricht, the Netherlands). The $m r p 14^{-/-}$mice, backcrossed $>10$ times to a C57BL/6 background were generated as described [21] and bred in the animal facility of the Academic Medical Centre (Amsterdam, the Netherlands). The $m r p 14^{-/-}$mice did not show any abnormalities in the healthy state $[21,22]$. Animal experiments were carried out in accordance with the Dutch Experiment on Animals Act and approved by the Animal Care and Use Committee of the University of Amsterdam (DIX100121AB).

\section{Design}

Mice were lightly anesthetised by inhalation of isoflurane (Abbot Laboratories, Queenborough, UK) and intranasally inoculated with a sublethal dose of $1 \times 10^{7}$ S. aureus USA300 (BK 11540) (kindly provided by Timothy J. Foster, Dept of Microbiology, Trinity College, Dublin, Ireland) in a $50 \mu \mathrm{l}$ saline solution $(\mathrm{n}=7-$ 8 per strain). This sublethal dose was determined in a previously described pilot study [23]. Mice were sacrificed $6,24,48$ or $72 \mathrm{~h}$ thereafter [23].

\section{Preparation of plasma samples and homogenates}

Collection and handling of samples were done as previously described [24, 25]. In brief, blood was drawn into heparinised tubes, bronchoalveolar lavage fluid (BALF) was obtained and organs were removed aseptically and homogenised in four volumes of sterile isotonic saline using a tissue homogeniser (Biospec Products, Bartlesville, UK). To determine bacterial loads, 10-fold dilutions were plated on blood agar plates and incubated at $37^{\circ} \mathrm{C}$ for $16 \mathrm{~h}$. Lung homogenates were prepared for immune assays as described previously $[24,25]$. 


\section{Bronchoalveolar lavage}

BALF was obtained as previously described [23]. The trachea and bronchi were exposed through a midline incision. The left main bronchus was ligated and the trachea was cannulated with a sterile 22-gauge Abbocath-T catheter (Abbott Laboratories, Sligo, Ireland). Unilateral, right-sided BAL was performed by instilling three $0.3 \mathrm{~mL}$ aliquots of sterile PBS. $0.7-0.9 \mathrm{~mL}$ of BALF was retrieved per mouse. Total cell numbers in BALF were counted using a Z2 Coulter particle count and size analyser (Beckman-Coulter, Inc., Miami, FL, USA). BALF differential cell counts were carried out on cytospin preparations stained with modified Giemsa stain (Diff-Quick; Baxter, McGraw Park, IL, USA).

Assays

Tumour necrosis factor (TNF)- $\alpha$, interleukin (IL)-1 $\beta$, IL-6, keratinocyte-derived chemokine (KC), macrophage inflammatory protein 2 (MIP-2) and E-selectin (all R\&D Systems, Minneapolis, MN, USA) and myeloperoxidase (MPO) (Hycult Biotechnology BV, Uden, the Netherlands) were measured using specific ELISAs according to manufacturers' recommendations. MRP8/14 [13] and nucleosomes [26] were measured as previously described.

\section{Histology}

Lung pathology scores were determined as described before $[24,25]$. In brief, lungs were harvested at the indicated time-points, fixed in $4 \%$ buffered formalin, and embedded in paraffin. $4 \mu \mathrm{m}$ sections were stained with haematoxylin and eosin (HE) and analysed by a pathologist blinded for groups as described earlier. To score lung inflammation and damage, the entire lung surface was analysed, with respect to the following parameters: bronchitis, oedema, interstitial inflammation, intra-alveolar inflammation, pleuritis, endothelialitis and percentage of the lung surface demonstrating confluent inflammatory infiltrate. Each parameter was graded $0-4$, with 0 being "absent" and 4 being "severe". The total pathology score was expressed as the sum of the score for all parameters. Granulocyte staining was done using fluorescein isothiocyanate-labelled rat anti-mouse Ly-6 mAb (Pharmingen, San Diego, CA, USA) as described previously [27]. Ly-6G expression in the lung tissue sections was quantified by digital image analysis [28]; the amount of Ly-6G positivity was expressed as a percentage of the total surface area. MRP8 and MRP14 staining in lung tissue were performed as previously described [21]. Staining of anti-citrullinated histone H3 (H3Cit) (Abcam, Cambridge, UK) of the lung tissue was done as previously described [29, 30].

\section{Statistical analysis}

Data are expressed as box and whisker plots depicting the smallest observation, lower quartile, median, upper quartile and largest observation. Differences between $m r p 14^{-/-}$and wildtype mice were analysed by Mann-Whitney U-test. Analyses were done using GraphPad Prism version 5.0 (Graphpad Software, San Diego, CA, USA). p-values $<0.05$ were considered statistically significant.

\section{Results}

MRP8/14 is released during staphylococcal pneumonia

To obtain insight into MRP8/14 expression during staphylococcal pneumonia, we measured MRP8/14 concentrations in BALF, lungs and plasma from uninfected wildtype mice and wildtype mice intranasally challenged with $101 \times 10^{7}$ S. aureus. MRP8/14 levels became detectable in BALF at $6 \mathrm{~h}$ post infection and increased during the course of the disease; the highest levels were found after $72 \mathrm{~h}$ (median $9 \mu \mathrm{g} \cdot \mathrm{mL}^{-1}$ ) (fig. 1a). In uninfected whole lung homogenates, MRP8/14 was detectable at low levels and was highly elevated at $6 \mathrm{~h}$ after infection (median $150 \mu \mathrm{g} \cdot \mathrm{mL}^{-1}$ at $24 \mathrm{~h}$ ) (fig. 1b). Plasma MRP8/14 levels were only minimally elevated at $6 \mathrm{~h}$ and reached basal levels shortly thereafter (data not shown).

To obtain insight into the cellular source of MRP8/14, we stained lung tissue slides obtained from naive and infected wildtype mice for MRP8 and MRP14. Naive lungs did not exhibit any staining of MRP8 or MRP14. After $6 \mathrm{~h}$, expression of both MRP8 and MRP14 increased strongly, which was still present $48 \mathrm{~h}$ after infection (fig. 1c and d).

\section{MRP14 deficiency minimally influences bacterial clearance during S. aureus pneumonia}

Earlier studies have shown that MRP8/14 inhibits staphylococcal growth in vitro [16] and in vivo $[17,18]$. To investigate a potential role for MRP8/14 in host defence during staphylococcal pneumonia, we harvested BAL fluid, lungs, livers and blood at $6,24,48$ or $72 \mathrm{~h}$ after infection and determined bacterial loads (fig. 2). Remarkably, MRP8/14 deficiency did not influence the clearance of $S$. aureus from the airways to a significant extent, although at a single time-point $(24 \mathrm{~h})$ bacterial burdens were modestly, but statistically significantly, higher in lung homogenates of $m r p 14^{-/-}$mice. Staphylococcal loads were similar in all other body compartments at all time-points in both mouse strains. These data suggest that MRP8/14 minimally contributes to bacterial clearance in a staphylococcal lung infection model. 

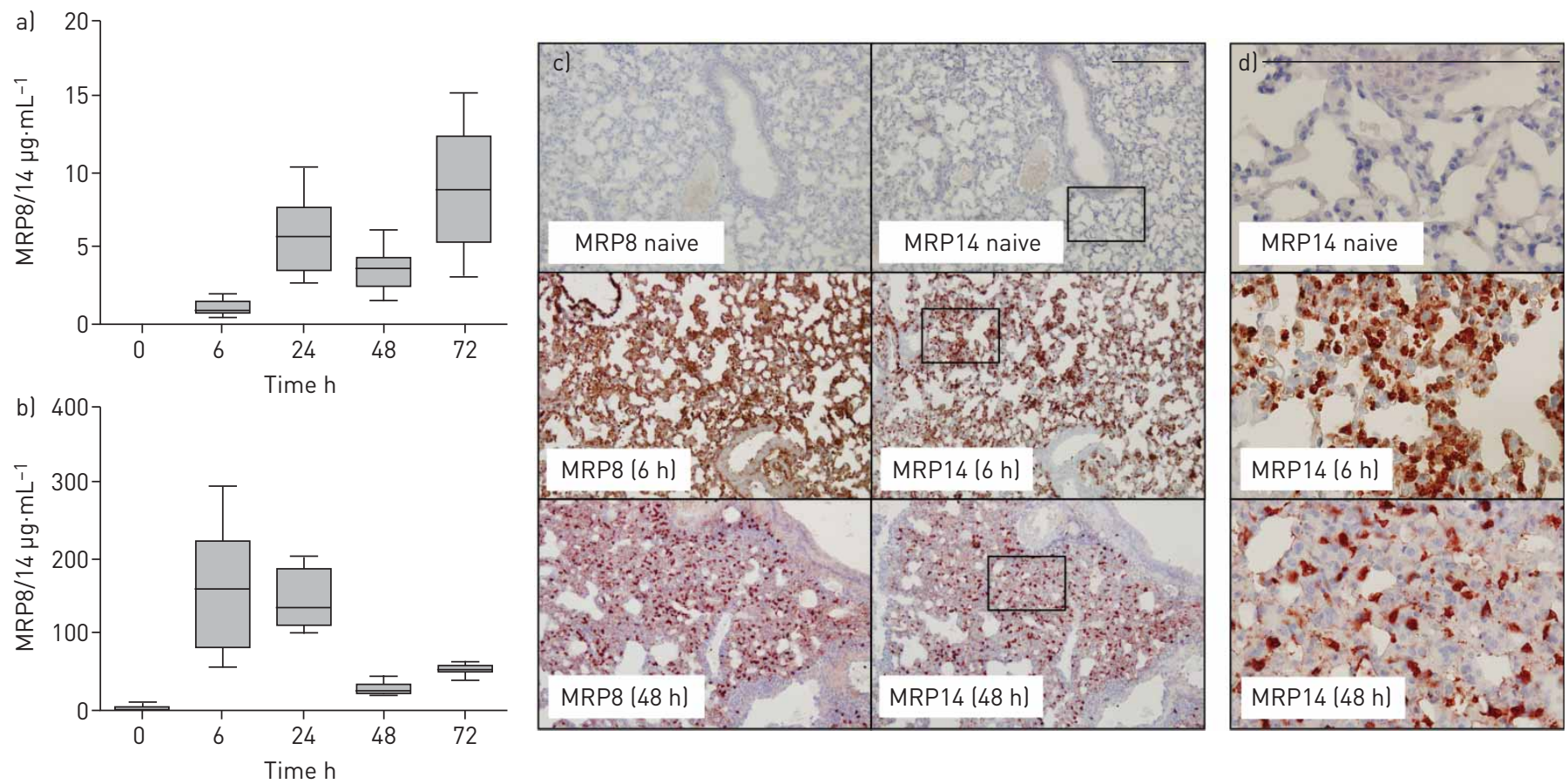

FIGURE 1 Murine staphylococcal pneumonia results in an increase in pulmonary myloid-related protein (MRP)8 and MRP14 (MRP8/14). The MRP8/14 levels in a) bronchoalveolar lavage fluid and b) whole lung homogenates in naive mice at 6,24, 48 and $72 \mathrm{~h}$ after intranasal Staphylococcus aureus infection $\left(1 \times 10^{7} \mathrm{CFU}\right)$. Data are expressed as box and whisker plots depicting the smallest observation, lower quartile, median, upper quartile and largest observation. c) MRP8 and MRP14 staining in lungs of naive mice and mice $6 \mathrm{~h}$ or $48 \mathrm{~h}$ after infection with S. aureus. d) A higher magnification of the boxed areas shown in c). Scale bars $=200 \mu \mathrm{m}$.
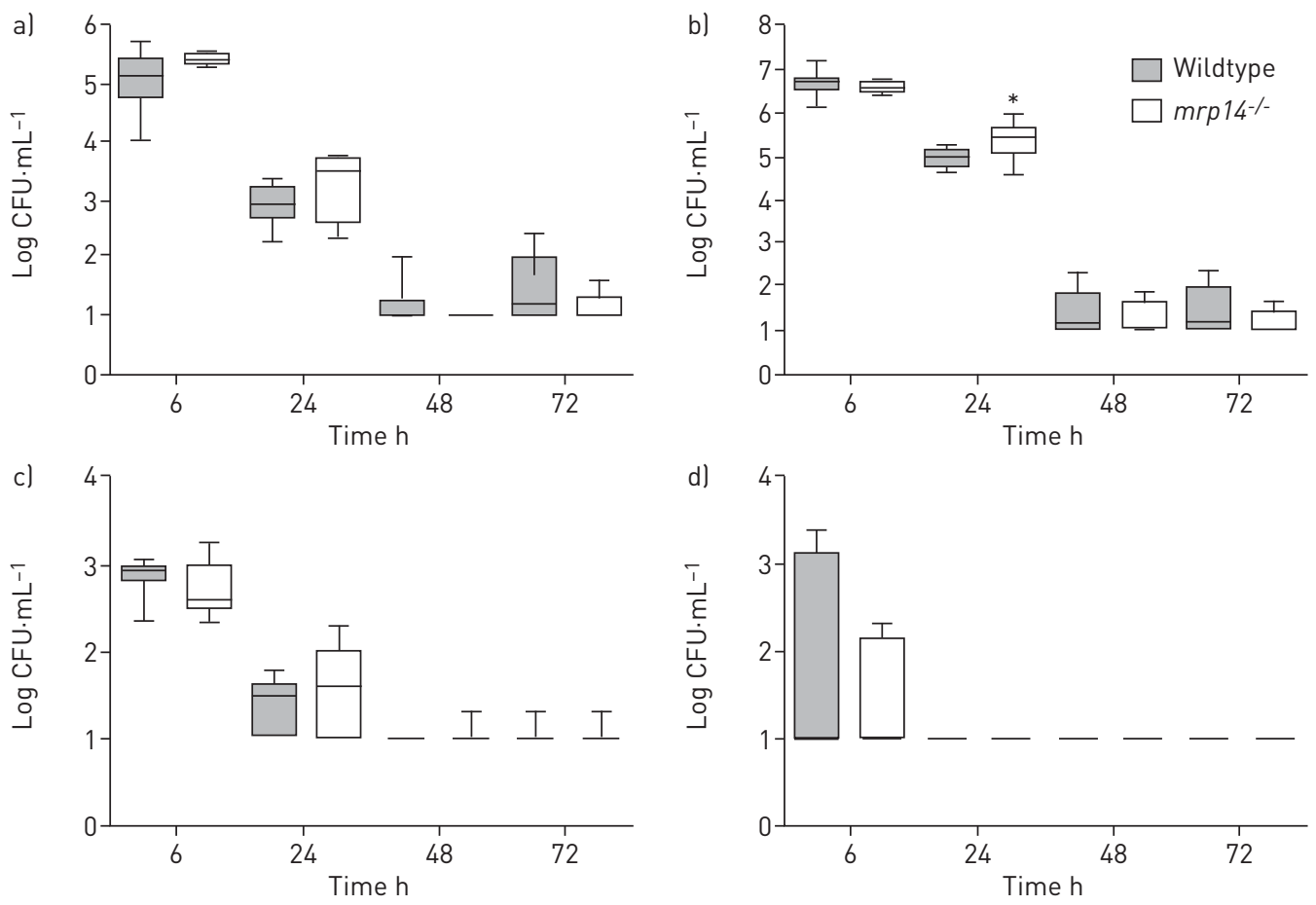

FIGURE 2 Myeloid-related protein (MRP)14-deficient $\left(\mathrm{mrp}_{14^{-/-}}\right)$mice display minimally enhanced bacterial outgrowth in Staphylococcus aureus pneumonia. Bacterial loads in a) bronchoalveolar lavage fluid, b) lung, c) liver and d) blood after intranasal inoculation of $1 \times 10^{7} \mathrm{CFU}$ of $S$. aureus in wildtype and $m r p 14^{-/-}$mice. Data are expressed as box and whisker plots depicting the smallest observation, lower quartile, median, upper quartile and largest observation (6-8 mice per group at each time-point). ${ }^{*}$ : $\mathrm{p}<0.05$, versus wildtype mice at the same time-point. 
MRP14 deficiency enhances early cytokine and chemokine release in S. aureus pneumonia Previous investigations have shown that MRP8/14 enhances the pro-inflammatory response of bone marrow derived macrophages, which correlates to lower plasma TNF- $\alpha$ levels in $m r p 14^{-1-}$ mice challenged with endotoxin [13]. To investigate the impact of loss of MRP14 on the inflammatory response in staphylococcal pneumonia, we measured cytokines (TNF- $\alpha$, IL-6 and IL-1 $\beta$ ) and chemokines (MIP-2 and $\mathrm{KC}$ ) in BALF wildtype and $m r p 14^{-/-}$mice at different time-points after infection with $S$. aureus via the airways (fig. 3a-e). The highest cytokine and chemokine levels were detected at $6 \mathrm{~h}$ after infection and diminished during the course of the disease. Surprisingly, $m r p 14^{-/-}$mice displayed increased levels of all measured cytokines and chemokines 6 and $24 \mathrm{~h}$ after infection (not significant for TNF- $\alpha$ and KC at $6 \mathrm{~h}$ ).

\section{MRP14 deficiency enhances lung pathology during S. aureus pneumonia}

We next analysed HE stained lung tissue slides to investigate the role of MRP8/14 in lung pathology in S. aureus pneumonia (fig. $4 \mathrm{a}-\mathrm{c}$ ). The total histological scores were semi-quantitatively scored according to the scoring system described in the Methods section. Already at $6 \mathrm{~h}$, all mice displayed signs of severe pulmonary inflammation. Loss of MRP8/14 resulted in aggravated lung pathology at this early time-point, with more advanced signs of interstitial inflammation and bronchitis. Lung pathology scores were still higher in $m r p 14^{-/-}$mice at $48 \mathrm{~h}$ after infection, with more endothelialitis, bronchitis and oedema. Histopathology analysis in this model is in correspondence to the cytokine data and suggests that MRP14 deficiency enhances pulmonary inflammation in murine $S$. aureus pneumonia.

To obtain additional insight into the inflammatory response at tissue level, we measured E-selectin levels in whole lung homogenates; the assay used does not discriminate between cell-bound and soluble E-selectin, and when performed on whole lung lysates can be used as a readout for endothelial cell activation [31]. Infection with $S$. aureus caused a brisk rise in whole lung E-selectin concentrations, peaking after $6 \mathrm{~h}$ and strongly decreasing thereafter (fig. 4d). Of notice, lung E-selectin levels were higher in $m r p 14^{-/-}$mice than in wildtype mice at all time-points, suggesting that MRP14 deficiency is associated with enhanced endothelial cell activation in the lungs.

\section{Impact of MRP14 deficiency on neutrophil influx and degranulation}

Staphylococcal pneumonia is associated with a massive influx of neutrophils, which is considered to play an important role in innate defence $[8,9,23]$. MRP8/14 has been implicated as an important mediator of neutrophil recruitment in various inflammatory conditions, including pneumonia [14,15]. We studied the recruitment of neutrophils into the lungs using various methods. Neutrophil numbers were similar in whole
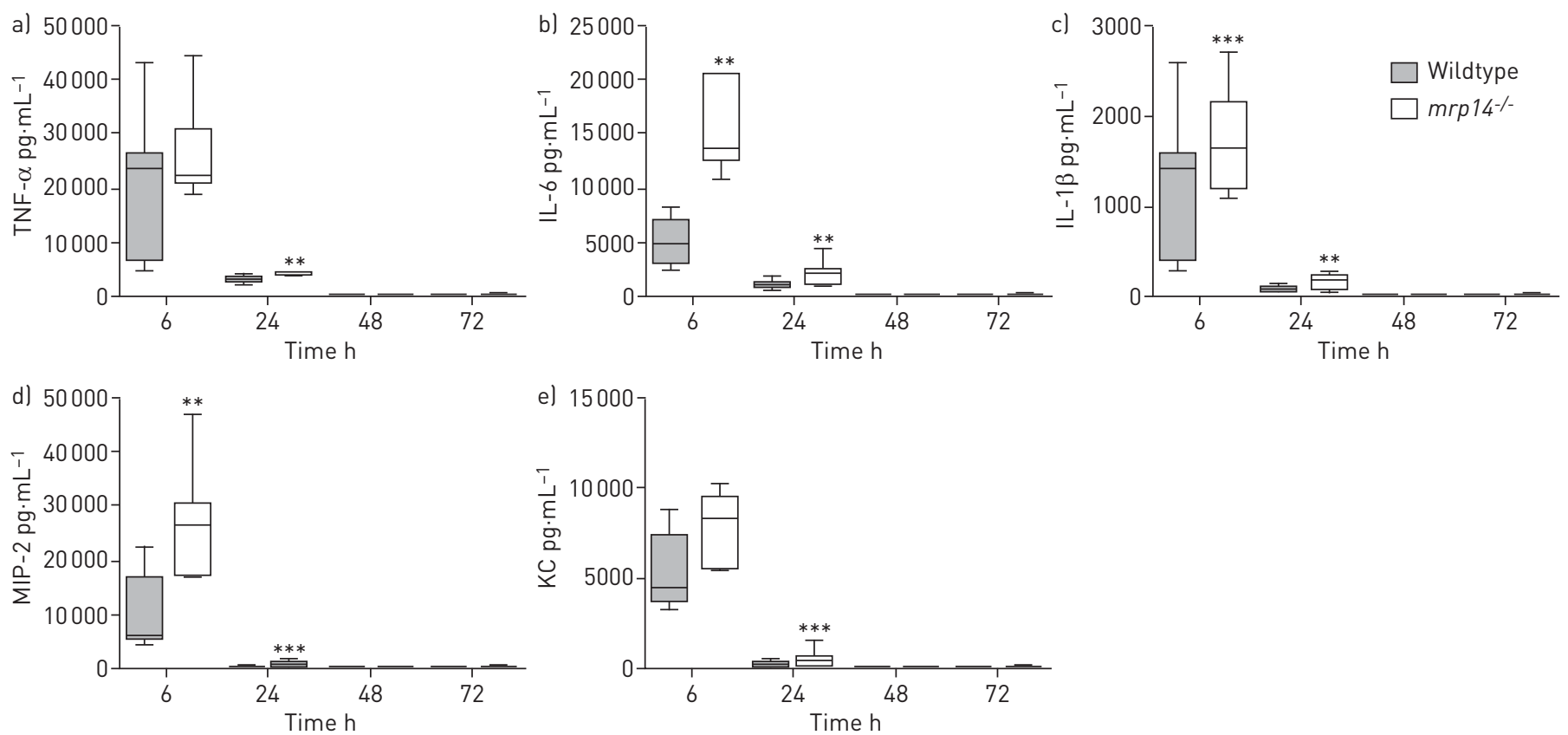

FIGURE 3 Myeloid-related protein (MRP)14-deficient $\left(\mathrm{mrp}^{-/-}\right)$mice display elevated cytokine levels in bronchoalveolar lavage fluid (BALF) during Staphylococcus aureus pneumonia. The levels of cytokines a) tumour necrosis factor (TNF)- $\alpha$, b) interleukin (IL)- 6 , and c) IL- $1 \beta$, in addition to the chemokines d) macrophage inflammatory protein (MIP)-2 and e) keratinocyte-derived chemokine (KC) in BALF are shown at 6, 24, 48 and $72 \mathrm{~h}$ after intranasal S. aureus infection in wildtype and $m r p 14^{-/-}$mice. Data are expressed as box and whisker plots depicting the smallest observation, lower quartile, median, upper quartile and largest observation (6-8 mice per group at each time-point). ${ }^{* *}$ : $\mathrm{p}<0.01{ }^{* * *}$ : $\mathrm{p}<0.001$ versus wildtype mice at the same time-point. 

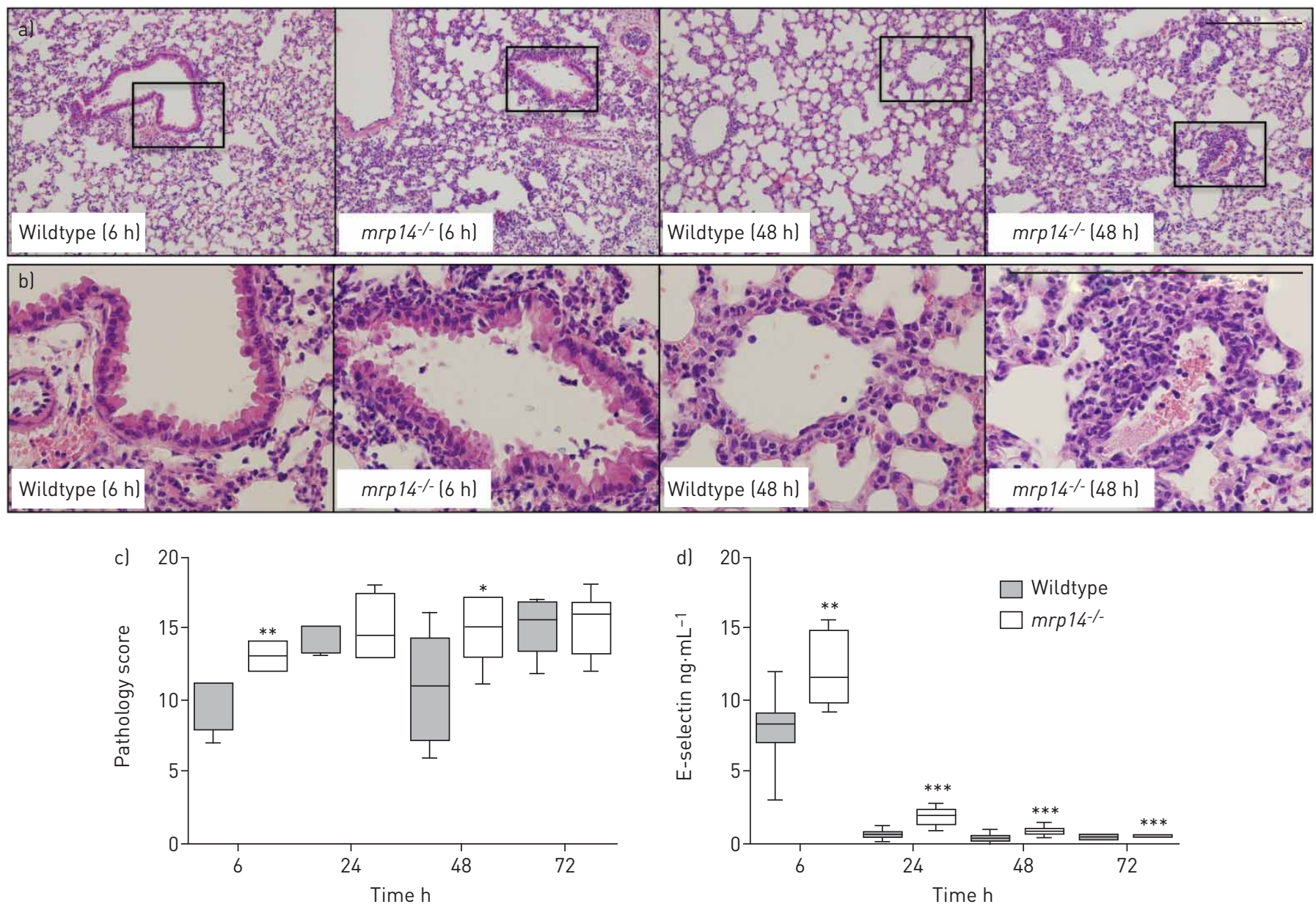

FIGURE 4 Myeloid-related protein (MRP)14-deficient $\left(\mathrm{mrp}^{-14^{--}}\right)$mice show enhanced lung pathology during Staphylococcus aureus pneumonia. a) Representative slides of lung haematoxylin and eosin staining of wildtype and $m r p 14^{-/-}$mice at $6 \mathrm{~h}$ and $48 \mathrm{~h}$ after intranasal S. aureus infection. b) Higher magnification of boxed areas shown in part a). c) Total pathology score, at indicated time-points post-infection in wildtype and $m r p 14^{-/-}$mice determined in accordance with the scoring system described in the Methods section. d) E-selectin levels were measured in lung homogenates as a marker for endothelial cell activation in wildtype and mrp14 $4^{-/-}$mice. Data are expressed as box and whisker plots depicting the smallest observation, lower quartile, median, upper quartile and largest observation (6-8 mice per group at each time-point). Scale bar $=200 \mu \mathrm{m} .{ }^{*}: \mathrm{p}<0.05,{ }^{* *}: \mathrm{p}<0.01,{ }^{* * *}: \mathrm{p}<0.001$, versus wildtype mice at the same time-point.

lungs as indicated by the numbers of Ly-6G positive cells in lung tissue slides prepared from $m r p 14^{-/-}$and wildtype mice between 6 and $72 \mathrm{~h}$ after infection (fig. 5a and b). Remarkably, pulmonary MPO concentrations, reflecting the number and degranulation of neutrophils in tissue, were higher in $m r p 14^{-/-}$ mice until $48 \mathrm{~h}$ post infection (fig. 5c). In BALF, neutrophil numbers were also similar in both mouse strains at 6 and $24 \mathrm{~h}$ after infection. However, at 48 and $72 \mathrm{~h}$, when the largest portion of bacteria had already been cleared, $m r p 14^{-/-}$mice showed significantly lower neutrophil numbers in BALF (fig. $5 \mathrm{~d}$ ). These data suggest differential effects of MRP14 deficiency on neutrophil recruitment in different lung compartments and on degranulation during S. aureus pneumonia.

\section{Role of MRP14 deficiency on NETs formation during S. aureus pneumonia}

Earlier investigations have shown that $S$. aureus is a potent inducer of NET release in vitro and in vivo [32, 33]. To determine the release of NETs in lungs during $S$. aureus pneumonia, we stained lung tissue slides from wildtype and $m r p 14^{-/-}$mice after staphylococcal infection for H3Cit, a marker for NETs [34]. Staining of H3Cit showed that both mouse strains released NETs in staphylococcal pneumonia. We next quantified nucleosome levels (a NET degradation product and a biomarker for NETs [35]) in BALF (fig. 6b). Despite the abundant presence of bacteria and neutrophils at $6 \mathrm{~h}$, nucleosomes were hardly detected in either of the mouse strains. At $24 \mathrm{~h}, \mathrm{mrp} 14^{-/-}$mice showed slightly higher levels of nucleosomes compared to the wildtype mice, while at later time-points $m r p 14^{-/-}$mice showed reduced nucleosome levels compared to the wildtype mice.

\section{Discussion}

MRSA pneumonia is associated with high morbidity and mortality rates in both immunocompromised patients [6] and in previously healthy individuals without recognised risk factors $[4,5]$. Staphylococci incite 


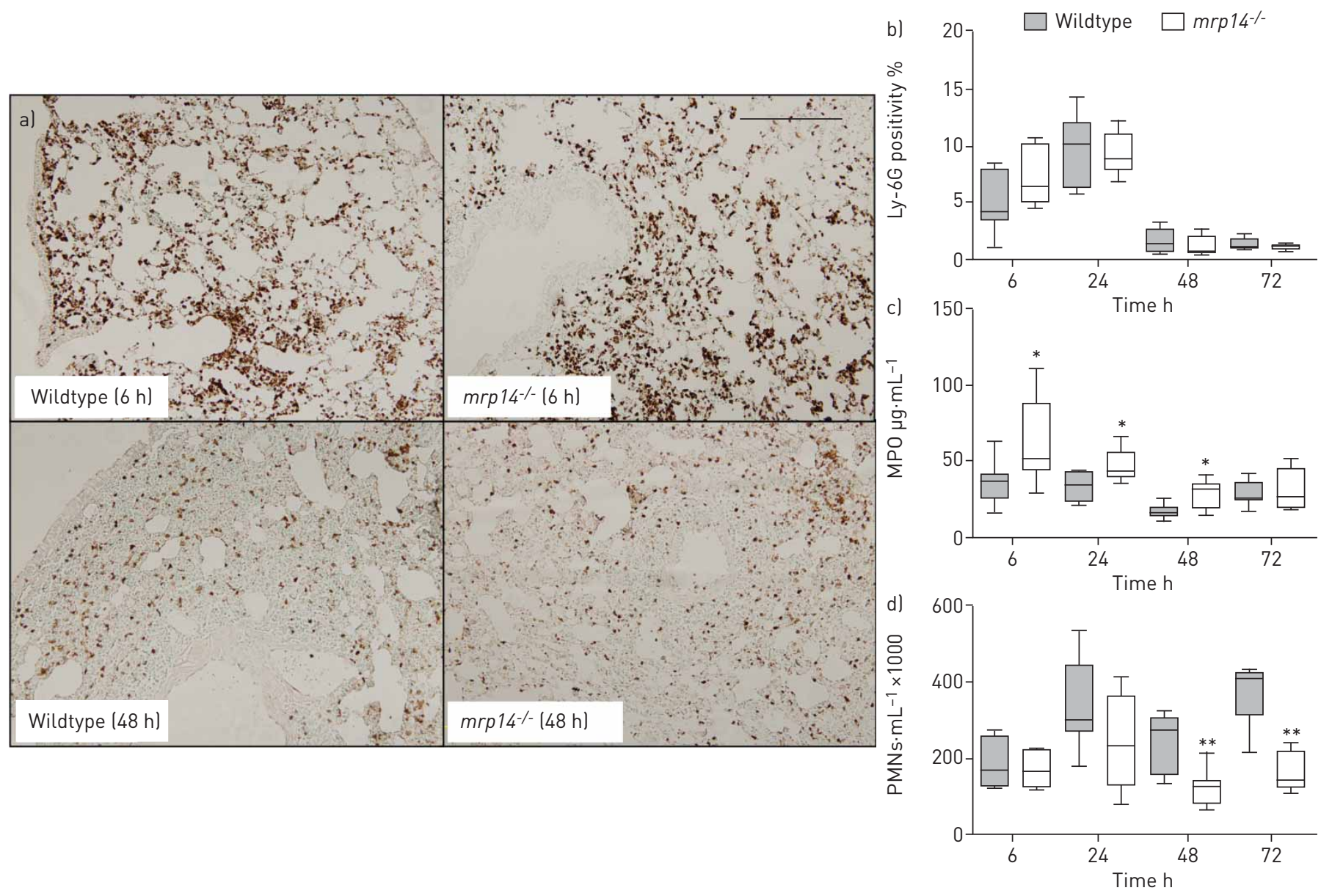

FIGURE 5 Myeloid-related protein (MRP)14-deficient $\left(\mathrm{mrp}^{-14^{--}}\right)$mice display diminished neutrophil transmigration to the bronchoalveolar space. a) Representative neutrophil staining (brown) of wildtype and $m r p 14^{-/-}$mice at $6 \mathrm{~h}$ and $48 \mathrm{~h}$ after induction of Staphylococcus aureus pneumonia. Quantitation of b) pulmonary Ly-6G positivity and c) myeloperoxidase (MPO) in whole lungs at $6,24,48$ and $72 \mathrm{~h}$ after infection in wildtype and $m r p 14^{-/-}$mice. d) Total polymorphonuclear neutrophil (PMN) numbers in bronchoalveolar lavage fluid at $6,24,48$ and $72 \mathrm{~h}$ after intranasal $S$. aureus infection in wildtype and mrp14 $14^{-/-}$mice. Data are expressed as box and whisker plots depicting the smallest observation, lower quartile, median, upper quartile and largest observation (6-8 mice per group at each time-point). Scale bar $=200 \mu \mathrm{m}$. ${ }^{*}: \mathrm{p}<0.05,{ }^{* *}: \mathrm{p}<0.01$ versus wildtype mice at the same time-point.
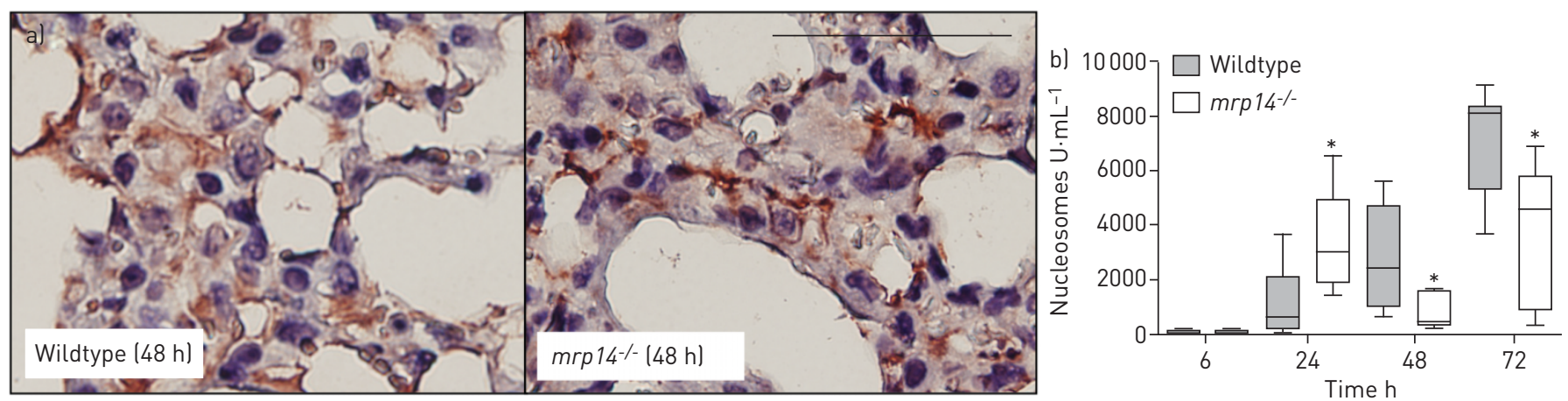

FIGURE 6 Wildtype and myeloid-related protein (MRP)14-deficient $\left(m r p 14^{-/-}\right)$mice release neutrophil extracellular traps (NETs) in the lungs after Staphylococcus aureus infection in the lungs. a) Staining of citrullinated H3 in lung tissue slides in wildtype and mrp $14^{-/-}$mice $48 \mathrm{~h}$ after intranasal S. aureus infection. b) Nucleosomes, a NET biomarker, was measured in wildtype and $m r p 14^{-1-}$ mice. Data are expressed as box and whisker plots depicting the smallest observation, lower quartile, median, upper quartile and largest observation $\left(6-8\right.$ mice per group at each time-point). Scale bar $=100 \mu \mathrm{m} .{ }^{*}: \mathrm{p}<0.05$ versus wildtype mice at the same time-point.

excessive inflammation upon lower airway infection, which may be perpetuated by alarmins [11], thereby contributing to lung injury and disease severity [7]. In the current study we aimed to determine the role of MRP8/14 in S. aureus induced pneumonia. Although MRP8/14 has been shown to facilitate the elimination of $S$. aureus in vitro [16] and after systemic infection in vivo $[17,18]$, the $m r p 14^{-/-}$mice surprisingly 
displayed a virtually unaltered capacity to clear $S$. aureus from their airways. Remarkably and in contrast to our hypothesis, MRP14 deficiency enhanced inflammation in lungs after intranasal S. aureus infection.

Human pneumonia is associated with local and systemic MRP8/14 release [36, 37], which correlates with findings in murine pneumonia caused by Klebsiella pneumoniae [20] or pneumococci [14, 37]. In accordance, in the current model of $S$. aureus pneumonia, MRP8/14 levels in plasma and lungs were also increased. The brisk rise of MRP8/14 at $6 \mathrm{~h}$ in lung homogenates may be a reflection of the strong pro-inflammatory stimulus induced by a high intranasal dose of $S$. aureus. The gradual increase of bronchoalveolar MRP8/14, which was at least 20 times higher than in Klebsiella pneumonia [20], might have been due to accumulation of released MRP8/14 after a massive early influx of activated neutrophils, which represent a major source of this protein $[38,39]$. We previously showed that MRP8 and the neutrophil marker Ly-6G have a similar expression in pneumococci infected lungs [37].

Despite high concentrations at the primary site of infection, MRP8/14 appeared to have an insignificant role in local bacterial control as staphylococcal loads were largely similar in $m r p 14^{-/-}$and wildtype mice. This finding is remarkable considering that MRP8/14 has been established as an antimicrobial protein in in vitro experiments, demonstrating reduced staphylococcal growth and virulence via the chelation of zinc and manganese $[16,17]$; additionally, the in vivo relevance of these data were shown in $m r p 14^{-1-}$ mice which displayed enhanced bacterial growth when challenged with $S$. aureus intravenously $[17,18]$. It has previously been demonstrated that MRP8/14 is abundantly present within abscesses [40] and has its greatest antimicrobial potential within these infectious environments [17, 18]. Distant abscess formation did not occur in our model of pneumonia. MRP8/14 may also inhibit bacterial growth via the formation of NETs $[19,20]$. We previously demonstrated that MRP8/14 is an important antimicrobial component within NETs to reduce $S$. aureus growth in vitro [20]. We here show that NETs are formed in S. aureus infected lungs of both mouse strains. Although wildtype and $m r p 14^{-/-}$neutrophils have a similar capacity to release NETs [19], we observed enhanced levels of nucleosomes, a NET biomarker [35], in BALF of $m r p 14^{-/-}$mice, $24 \mathrm{~h}$ after infection, which may be a reflection of the enhanced pro-inflammatory state in these mice at this timepoint. The enhanced nucleosome levels in wildtype mice at 48 and $72 \mathrm{~h}$ most likely reflect the increased number of neutrophils in the bronchoalveolar space. Interestingly, nucleosome levels were only present when the majority of bacteria were cleared. Previous investigations have already shown that NETosis may occur in vivo in the presence of $S$. aureus [33]. However, not all neutrophils form NETs and only when bacteria are too large to be phagocytised, e.g. upon formation of large staphylococcal aggregates [41]. We speculate that a large portion of the inoculated bacteria was easily phagocytised by neutrophils, which, therefore, did not form NETs at this early time-point [41]. The fact that nucleosomes were highly present in late stage disease may be due to NETosis in response to remaining bacteria that are not easily phagocytised and due to accumulation of NET degradation products. Together these data suggest that MRP8/14 (within or outside of NETs) has a limited antibacterial role in staphylococcal pneumonia.

While previous studies found pro-inflammatory effects of MRP8/14 under various pathological conditions [13], we were unable to demonstrate such a role for MRP8/14 in S. aureus pneumonia. In contrast, we unexpectedly observed higher cytokine and chemokine levels in lungs and more advanced signs of histopathology in $\mathrm{mrp} 14^{-/-}$mice. This pro-inflammatory phenotype of $\mathrm{mrp} 14^{-/-}$mice is unlikely explained by differences in bacterial loads, considering that many dissimilarities were already present at $6 \mathrm{~h}$ after infection (when all bacterial counts were similar) and considering the only very modest variance in lung bacterial loads at $24 \mathrm{~h}$. It is also not explained by different baseline cytokine and chemokine levels, as these are similar in BALF from naive wildtype and $m r p 14^{-/-}$mice [42]. Recent studies demonstrated that $m r p 14^{-/}$mice have an enhanced maturation state of dendritic cells [43, 44], and that these matured dendritic cells release more cytokines [44]. Notably, dendritic cells contribute to cytokine production during S. aureus pneumonia [45]. Further studies are needed to establish a possible role of these immune cells in the hyperinflammatory phenotype of $m r p 14^{-/-}$mice in this model.

Several animal studies have implicated MRP8/14 as a mediator of neutrophil recruitment $[14,15]$. In the current study, we found similar neutrophil numbers at 6 and $24 \mathrm{~h}$ after infection in BALF and lung tissue of wildtype and $m r p 14^{-/-}$mice. However, at 48 and $72 \mathrm{~h}$ after infection; when the bulk of bacteria had been cleared from the lungs, mrp $14^{-1-}$ mice displayed markedly diminished neutrophil numbers in BALF, suggesting that MRP8/14 may play a role to attract neutrophils within the bronchoalveolar compartment but that this lack of a chemotactic effect in $\mathrm{mrp} 14^{-/-}$mice may be compensated for by other mechanisms induced by viable bacteria at early time-points. Remarkably, MRP8/14 did not influence neutrophil numbers in lung tissue, as shown by Ly-6G staining, suggesting that the high MRP8/14 levels in BALF at later time-points impacts on neutrophil transmigration into the bronchoalveolar space. Indeed, taking the dilution factor introduced by the BAL procedure into account, BALF MRP8/14 levels were higher than those in lung tissue at 48 and $72 \mathrm{~h}$ post infection. Despite similar neutrophil numbers in whole lungs, pulmonary MPO levels were increased in $\mathrm{mrp}_{14^{-/-}}$mice, suggesting enhanced degranulation of 
neutrophils, which may be secondary to the enhanced inflammatory state. Notably, MRP14 deficiency did not impact on viability of neutrophils, or on neutrophil expression of adhesion receptors or respiratory burst in previous studies [21,22].

Previous studies have shown that endothelial cell activation, as shown by E-selectin levels, is enhanced by MRP8/14 [15, 46]. Hence, we reason that the increased E-selectin levels in total lung homogenates of mrp $14^{-1-}$ mice may be due to the enhanced inflammatory state as well.

A limitation of our study is that mice are relatively resistant to $S$. aureus infection compared to humans, at least in part due to the inability of this bacterium to release iron from murine haemoglobin [47]. To model clinical pneumonia, mice have to be intranasally inoculated with high staphylococcal doses [7]. Extrapolation of our results to the human situation should, therefore, be done with caution. In addition, our study does not provide insight into which cell-type drives the phenotype of $\mathrm{mrp} 14^{-/-}$mice. Although neutrophils are likely to be the most important, adoptive transfer and/or bone marrow transplantation studies are required to obtain proof for this. While the former approach is technically challenging in mice, the latter approach might be less feasible considering incomplete replacement of alveolar macrophages [48].

In conclusion, we here document that $S$. aureus pneumonia is associated with accumulating MRP8/14 in the lungs, which promotes neutrophil transmigration to the bronchoalveolar space but has a limited role in clearance of bacteria from the airways. MRP14 deficiency is associated with enhanced pulmonary inflammation, neutrophil degranulation and endothelial cell activation in $S$. aureus pneumonia, suggesting that MRP8/14 may serve an unexpected lung protective role in severe $S$. aureus pneumonia.

\section{Acknowledgements}

We thank Timothy J. Foster (Dept of Microbiology, Trinity College, Dublin, Ireland) for the Staphylococcus aureus strain in these experiments.

\section{References}

1 Chambers HF, Deleo FR. Waves of resistance: Staphylococcus aureus in the antibiotic era. Nat Rev Microbiol 2009; 7: 629-641.

2 Chastre J, Blasi F, Masterton RG, et al. European perspective and update on the management of nosocomial pneumonia due to methicillin-resistant Staphylococcus aureus after more than 10 years of experience with linezolid. Clin Microbiol Infect 2014; 20: Suppl. 4, 19-36.

3 David MZ, Daum RS. Community-associated methicillin-resistant Staphylococcus aureus: epidemiology and clinical consequences of an emerging epidemic. Clin Microbiol Rev 2010; 23: 616-687.

4 Gillet Y, Issartel B, Vanhems P, et al. Association between Staphylococcus aureus strains carrying gene for Panton-Valentine leukocidin and highly lethal necrotising pneumonia in young immunocompetent patients. Lancet 2002; 359: 753-759.

5 Francis JS, Doherty MC, Lopatin U, et al. Severe community-onset pneumonia in healthy adults caused by methicillin-resistant Staphylococcus aureus carrying the Panton-Valentine leukocidin genes. Clin Infect Dis 2005; 40: 100-107.

6 Rubinstein E, Kollef MH, Nathwani D. Pneumonia caused by methicillin-resistant Staphylococcus aureus. Clinic Infect Dis 2008; 46: Suppl. 5, S378-S385.

7 Parker D, Prince A. Immunopathogenesis of Staphylococcus aureus pulmonary infection. Semin Immunopathol 2012; 34: 281-297.

8 Bartlett AH, Foster TJ, Hayashida A, et al. $\alpha$-toxin facilitates the generation of CXC chemokine gradients and stimulates neutrophil homing in Staphylococcus aureus pneumonia. J Infect Dis 2008; 198: 1529-1535.

9 Diep BA, Chan L, Tattevin P, et al. Polymorphonuclear leukocytes mediate Staphylococcus aureus Panton-Valentine leukocidin-induced lung inflammation and injury. Proc Natl Acad Sci USA 2010; 107: 5587-5592.

10 Rigby KM, DeLeo FR. Neutrophils in innate host defense against Staphylococcus aureus infections. Semin Immunopathol 2012; 34: 237-259.

11 Bianchi ME. DAMPs, PAMPs and alarmins: all we need to know about danger. J Leukoc Biol 2007; 81: 1-5.

12 Ehrchen JM, Sunderkotter C, Foell D, et al. The endogenous Toll-like receptor 4 agonist S100A8/S100A9 (calprotectin) as innate amplifier of infection, autoimmunity, and cancer. J Leukoc Biol 2009; 86: 557-566.

13 Vogl T, Tenbrock K, Ludwig S, et al. Mrp8 and Mrp14 are endogenous activators of Toll-like receptor 4, promoting lethal, endotoxin-induced shock. Nat Med 2007; 13: 1042-1049.

14 Raquil MA, Anceriz N, Rouleau P, et al. Blockade of antimicrobial proteins S100A8 and S100A9 inhibits phagocyte migration to the alveoli in streptococcal pneumonia. J Immunol 2008; 180: 3366-3374.

15 Viemann D, Strey A, Janning A, et al. Myeloid-related proteins 8 and 14 induce a specific inflammatory response in human microvascular endothelial cells. Blood 2005; 105: 2955-2962.

16 Damo SM, Kehl-Fie TE, Sugitani N, et al. Molecular basis for manganese sequestration by calprotectin and roles in the innate immune response to invading bacterial pathogens. Proc Natl Acad Sci USA 2013; 110: 3841-3846.

17 Kehl-Fie TE, Chitayat S, Hood MI, et al. Nutrient metal sequestration by calprotectin inhibits bacterial superoxide defense, enhancing neutrophil killing of Staphylococcus aureus. Cell Host Microbe 2011; 10: 158-164.

18 Corbin BD, Seeley EH, Raab A, et al. Metal chelation and inhibition of bacterial growth in tissue abscesses. Science 2008; 319: 962-965.

19 Urban CF, Ermert D, Schmid M, et al. Neutrophil extracellular traps contain calprotectin, a cytosolic protein complex involved in host defense against Candida albicans. PLoS Pathog 2009; 5: e1000639. 
20 Achouiti A, Vogl T, Urban CF, et al. Myeloid-related protein-14 contributes to protective immunity in Gram-negative pneumonia derived sepsis. PLoS Pathog 2012; 8: e1002987.

21 Manitz MP, Horst B, Seeliger S, et al. Loss of S100A9 (MRP14) results in reduced interleukin-8-induced CD11b surface expression, a polarized microfilament system, and diminished responsiveness to chemoattractants in vitro. Mol Cell Biol 2003; 23: 1034-1043.

22 Hobbs JA, May R, Tanousis K, et al. Myeloid cell function in MRP-14 (S100A9) null mice. Mol Cell Biol 2003; 23: 2564-2576.

23 Achouiti A, van der Meer AJ, Florquin S, et al. High-mobility group box 1 and the receptor for advanced glycation end products contribute to lung injury during Staphylococcus aureus pneumonia. Crit Care 2013; 17: R296.

24 Rijneveld AW, Weijer S, Florquin S, et al. Thrombomodulin mutant mice with a strongly reduced capacity to generate activated protein $\mathrm{C}$ have an unaltered pulmonary immune response to respiratory pathogens and lipopolysaccharide. Blood 2004; 103: 1702-1709.

25 Wieland CW, van Lieshout MH, Hoogendijk AJ, et al. Host defence during Klebsiella pneumonia relies on haematopoietic-expressed Toll-like receptors 4 and 2. Eur Respir J 2011; 37: 848-857.

26 Zeerleder S, Stephan F, Emonts M, et al. Circulating nucleosomes and severity of illness in children suffering from meningococcal sepsis treated with protein C. Crit Care Med 2012; 40: 3224-3229.

27 Achouiti A, de Vos AF, de Beer R, et al. Limited role of the receptor for advanced glycation end products during Streptococcus pneumoniae bacteremia. J Innate Immun 2013; 5: 603-612.

28 Lammers AJ, de Porto AP, Florquin S, et al. Enhanced vulnerability for Streptococcus pneumoniae sepsis during asplenia is determined by the bacterial capsule. Immunobiology 2011; 216: 863-870.

29 Abrahamian FM, Moran GJ. Methicillin-resistant Staphylococcus aureus infections. N Engl J Med 2007; $357: 2090$.

30 de Stoppelaar SF, van 't Veer C, Claushuis TA, et al. Thrombocytopenia impairs host defense in Gram-negative pneumonia derived sepsis. Blood 2014; 124: 3781-3790.

31 Hoogerwerf JJ, Leendertse M, Wieland CW, et al. Loss of suppression of tumorigenicity 2 (ST2) gene reverses sepsis-induced inhibition of lung host defense in mice. Am J Respir Crit Care Med 2011; 183: 932-940.

32 Pilsczek FH, Salina D, Poon KK, et al. A novel mechanism of rapid nuclear neutrophil extracellular trap formation in response to Staphylococcus aureus. J Immunol 2010; 185: 7413-7425.

33 Yipp BG, Petri B, Salina D, et al. Infection-induced NETosis is a dynamic process involving neutrophil multitasking in vivo. Nat Med 2012; 18: 1386-1393.

34 Chen G, Zhang D, Fuchs TA, et al. Heme-induced neutrophil extracellular traps contribute to the pathogenesis of sickle cell disease. Blood 2014; 123: 3818-3827.

35 Thomas GM, Carbo C, Curtis BR, et al. Extracellular DNA traps are associated with the pathogenesis of TRALI in humans and mice. Blood 2012; 119: 6335-6343.

36 van Zoelen MA, Vogl T, Foell D, et al. Expression and role of myeloid-related protein-14 in clinical and experimental sepsis. Am J Respir Crit Care Med 2009; 180: 1098-1106.

37 Achouiti A, Vogl T, Endeman H, et al. Myeloid-related protein-8/14 facilitates bacterial growth during pneumococcal pneumonia. Thorax 2014; 69: 1034-1042.

38 Edgeworth J, Gorman M, Bennett R, et al. Identification of p8,14 as a highly abundant heterodimeric calcium binding protein complex of myeloid cells. J Biol Chem 1991; 266: 7706-7713.

39 Goyette J, Geczy CL. Inflammation-associated S100 proteins: new mechanisms that regulate function. Amino Acids 2011; 41: 821-842

40 Clohessy PA, Golden BE. Calprotectin-mediated zinc chelation as a biostatic mechanism in host defence. Scand J Immunol 1995; 42: 551-556.

41 Branzk N, Lubojemska A, Hardison SE, et al. Neutrophils sense microbe size and selectively release neutrophil extracellular traps in response to large pathogens. Nat Immunol 2014; 15: 1017-1025.

42 Kuipers MT, Vogl T, Aslami H, et al. High levels of S100A8/A9 proteins aggravate ventilator-induced lung injury via TLR4 signaling. PLoS One 2013; 8: e68694.

43 Petersen B, Wolf M, Austermann J, et al. The alarmin Mrp8/14 as regulator of the adaptive immune response during allergic contact dermatitis. EMBO J 2013; 32: 100-111.

44 Averill MM, Barnhart S, Becker L, et al. S100A9 differentially modifies phenotypic states of neutrophils, macrophages, and dendritic cells: implications for atherosclerosis and adipose tissue inflammation. Circulation 2011; 123: 1216-1226.

45 Parker D, Prince A. Staphylococcus aureus induces type I IFN signaling in dendritic cells via TLR9. J Immunol 2012; 189: 4040-4046.

46 Vogl T, Eisenblatter M, Voller T, et al. Alarmin S100A8/S100A9 as a biomarker for molecular imaging of local inflammatory activity. Nat Commun 2014; 5: 4593.

47 Pishchany G, McCoy AL, Torres VJ, et al. Specificity for human hemoglobin enhances Staphylococcus aureus infection. Cell Host Microbe 2010; 8: 544-550.

48 Taut K, Winter C, Briles DE, et al. Macrophage turnover kinetics in the lungs of mice infected with Streptococcus pneumoniae. Am J Respir Cell Mol Biol 2008; 38: 105-113. 